\title{
A novel 3D modelling and simulation technique in thermotherapy predictive analysis on biological tissue
}

\author{
F. Fanjul-Vélez ${ }^{* a}$, J. L. Arce-Diego ${ }^{* a}$, Oleg G. Romanov ${ }^{b}$, Alexei L. Tolstik ${ }^{\mathrm{b}}$ \\ aApplied Optical Techniques Group, TEISA Department, University of Cantabria, Av. de los Castros \\ S/N, 39005 Santander (Spain) \\ ${ }^{\mathrm{b}}$ Laser Physics and Spectroscopy Department, Belarusian State University, 4, Fr. Nezalezhnasti av., \\ 220050 Minsk (Belarus)
}

\begin{abstract}
Optical techniques applied to biological tissue allow the development of new tools in medical praxis, either in tissue characterization or treatment. Examples of the latter are Photodynamic Therapy (PDT) or Low Intensity Laser Treatment (LILT), and also a promising technique called thermotherapy, that tries to control temperature increase in a pathological tissue in order to reduce or even eliminate pathological effects. The application of thermotherapy requires a previous analysis in order to avoid collateral damage to the patient, and also to choose the appropriate optical source parameters. Among different implementations of opto-thermal models, the one we use consists of a three dimensional Beer-Lambert law for the optical part, and a bio-heat equation, that models heat transference, conduction, convection, radiation, blood perfusion and vaporization, solved via a numerical spatial-temporal explicit finite difference approach, for the thermal part. The usual drawback of the numerical method of the thermal model is that convergence constraints make spatial and temporal steps very small, with the natural consequence of slow processing. In this work, a new algorithm implementation is used for the bio-heat equation solution, in such a way that the simulation time decreases considerably. Thermal damage based on the Arrhenius integral damage is also considered.
\end{abstract}

Keywords: optical treatment, thermotherapy, bio-heat equation, finite difference numerical method, thermal damage

\section{INTRODUCTION}

The field of medicine is being helped by new methods and techniques coming from advances in technology. Among them, the contribution of the branch of optics supplies procedures and tools mainly in two aspects: characterization and treatment of biological tissue. Having to do with the former, optical techniques provide improved high resolution images obtained in a non-contact, non-invasive and non-ionizing way, like in Optical Coherence Tomography (OCT) [1] or in Fluorescence Spectroscopy [2]. The latter application of optics in biomedicine tries to mitigate or even suppress pathological effects of diseases by means of optical radiation. The variability of such therapies is high, and we could mention examples like Photodynamic Therapy (PDT) [3], Low Intensity Laser Therapy (LILT) or Hyperthermia [4].

PDT or Hyperthermia are intended to destroy some amount of pathological tissue, usually in patients suffering from cancer. On the contrary, there are another applications of optical radiation to tissues in which the main objective is the recovery of the affected tissue with no destruction, such in LILT or in the so-called thermotherapy. This last optical treatment is intended to increase temperature of a pathological tissue in a controlled way, so as to provoke a reduction of the effects of the disease, and in some cases to restore the normal behaviour of the affected part [5]. Temperature increase should usually be low, so as not to produce a harmful effect on the tissue under treatment.

The security margins required in medical praxis make the procedure control fundamental. Modelling light-tissue interaction allows the possibility to predict the tissue behaviour when it is irradiated. In the particular case of thermotherapy, it is important to know the temperature distribution in the pathological tissue from the optical source parameters. There are some approaches that try to deal with this problem [2], and they usually divide the problem in an optical and a thermal model, that are of course interlaced.

* ffanjul@teisa.unican.es; jlarce@ateisa.unican.es; phone +34 94220 15 45; fax +34 94220 18 73; www.teisa.unican.es/toa

Therapeutic Laser Applications and Laser-Tissue Interactions III, edited by Alfred Vogel, Proc. of SPIE-OSA Biomedical Optics, SPIE Vol. 6632, 66320D, @ 2007 SPIE-OSA $1605-7422 / 07 / \$ 18$ 
The complexity of the models used make it necessary to implement numerical methods in order to get a solution for the differential equations that govern optical and thermal interactions. For the optical part, results can be obtained with a reasonable accuracy in a reasonable time when using for instance a Radiation Transfer Theory (RTT) model or a BeerLambert law [2]. However, for the thermal part a bio-heat equation is usually considered, solved via numerical methods like explicit finite difference. This procedure makes the simulation procedure slow, and usually does not consider effects such as spatial or temporal mesh dynamic refinement, or the increase of the analysed area as a function of temperature spatial changes when approaching boundaries.

In this work, a complete thermo-optical model is presented and programmed. The optical part is modelled by means of a three dimensional Beer-Lambert law, and thermal interactions are simulated via a bio-heat equation, that takes into account heat transference, conduction, convection, radiation, blood perfusion and vaporization. This bio-heat equation is solved by means of a novel 3D two steps modified Du Fort-Frankel method, that reduces considerably the computation time and allows a better prediction of temperature distribution. Adaptive mesh refinement procedures both in time and spatial coordinates, that give us the possibility to cover all heating area and to calculate transients of temperature distribution with the use of a modern PC in a reasonable CPU time, are included. Such a speed improvement allow a quicker thermotherapy prediction and so the consideration of several sources can be taken in a reasonable time period. The complete model can be used for concrete treatment proposals, as a way of predicting treatment effects and consequently decide which optical source parameters are appropriate for the specific disease, mainly wavelength and optical power, with reasonable security margins in the process, and in a appreciably minor computational time.

\section{LIGHT-TISSUE INTERACTION MODELLING}

One of the fundamental points when trying to predict thermotherapy treatment procedures is to adequately model light-tissue interaction. When a tissue is irradiated by a light source, effects like photodisruption or photochemical interaction may appear, depending mainly on source power and pulse duration. Thermal interaction is shown approximately when irradiance is from $\mathrm{mW} / \mathrm{cm}^{2}$ to around hundreds of $W / \mathrm{cm}^{2}$, with pulse durations of the order of ms to continuous wave $(\mathrm{CW})$ and exposition goes from hundreds of ms until minutes or tens of minutes. Under these conditions a temperature increase is appreciated and so the therapeutic process takes place.

Modelling the thermal process in light-tissue interaction requires on one hand, an adequately consideration of optical propagation in tissue and on the other hand, a model for heat propagation of energy generated by the previous irradiation. In next sections, these two aspects are analysed and the proposed integrated thermo-optical model is presented. Attention is paid also to the prediction of thermal damage in tissue, of great importance when dealing with methods related with medical procedures, due to security margins and collateral harmful effects.

\subsection{Light propagation in tissue}

Light propagation in tissue is a complex problem, mainly due to the fact that biological tissues are heterogeneous media and, as a consequence, their optical characteristics are different in principle in each individual point. However, some assumptions can be made in order to make the problem easier. In this way, the heterogeneous medium is usually supposed to be macroscopically homogeneous, and so global optical properties can be extracted from it. These averaged properties can be used in models that approach reasonably good real tissue behaviour.

There are several models that approximate light propagation in tissue. If the geometries considered are simple, like spheres or cylinders, it is possible even to apply Maxwell equations to the problem and to obtain light energy distribution inside [6]. Of course when dealing with real biological tissue this situation is not going to appear, and a more general model is preferred. Kubelka-Munk [2] model considers a two fluxes approach in the irradiated tissue, and there are models that use even seven fluxes [7]. The Radiation Transport Theory (RTT) consists of a three dimensional differential equation, that takes into account refraction index mismatch, absorption and scattering [2]. On the contrary, others reduce the problem to a mainly one-dimensional model based on a simple exponential [8], and obtain reasonably good results.

In our case, we intend to use a three dimensional model of light propagation in tissue that allows us to know the energy deposition in each point of our tissue. Among the various possibilities, we chose a three dimensional BeerLambert law, that is expressed by the following equation: 


$$
Q_{s}=\alpha I_{0} e^{-\alpha z} e^{-s\left(x^{2}+y^{2}\right)},
$$

where $I_{0}$ is the incident irradiance $\left(W / \mathrm{cm}^{2}\right), \alpha$ is the absorption depth coefficient $\left(\mathrm{cm}^{-1}\right)$ in depth, and $s\left(\mathrm{~cm}^{-2}\right)$ takes into account the beam spatial gaussian radius $r_{0}\left(s=1 / r_{0}^{2}\right)$. The resulting parameter $Q_{s}$ shows the energy per volume unit $\left(\mathrm{W} / \mathrm{cm}^{3}\right)$ delivered by the optical source in the tissue.

The energy distribution will be the heat source for the subsequent thermal analysis that will be carried out in next subsection.

\subsection{Thermal model of light irradiated tissue}

Once we have the optical distribution in tissue, and knowing that it is suffering from a thermal interaction, we must construct a thermal model. The main objective of this section is the achievement of a model that allows us to predict temperature in each point and in each time, due to the fact that exposition time becomes a crucial variable to be taken into account.

Heat distribution in any material is related with heat transference processes. These are conduction, that treats heat transmission in a solid; convection, which deals with heat interchange between a solid and a fluid in contact with it; and radiation, that has to do with the infrared radiation that any body at nonzero temperature emits naturally [9]. All these effects have been studied for a long time. In the particular case of biological tissue, heat transference mechanism follows the same approaches as a general material, except from the consideration of blood flow. The existence of perfusion in in vivo biological tissue makes it necessary to account for it in the thermal model, due to the fact that this blood flow provokes convection with the surrounding tissue, and so a temperature decrease appears.

Heat transmission mechanisms are usually treated by including them in a so-called bio-heat equation. This is a spatial-temporal differential equation that models temperature distribution in tissue. As usual, there are several approaches to this aim, like the one developed by Pennes, by Chen and Holmes, the Weinbaum, Jiji and Lemons (WJL) model or the Wissler model [10]. They all are based on a balanced equation that shows energy fluxes around the tissue:

$$
Q_{\text {gain }}=Q_{\text {storage }}+Q_{\text {loss }}+W \text {, }
$$

where $\mathrm{W}$ indicates the heat internally generated, that is considered zero in our case. From this expression, a bio-heat equation can be constructed:

$$
\rho c \frac{\delta T}{\delta t}=k \nabla^{2} T+Q_{s}+Q_{P} .
$$

In this equation, $\rho$ is the tissue density; $c$ stands for specific heat $(J / \mathrm{kg} \cdot \mathrm{K})$; T is the tissue temperature in $\mathrm{K}$; $Q_{S}$ contains the energy delivered by the optical source; and $Q_{P}$ represents the perfusion term applicable when dealing with in vivo biological tissues:

$$
Q_{p}=-\rho_{b} c_{b} w_{b}\left(T_{v e n}-T_{a r t}\right),
$$

where subindex $\mathrm{b}$ stands for blood, $w$ is the perfusion blood rate $\left(\mathrm{m}_{b}^{3} / \mathrm{m}^{3} \cdot \mathrm{s}\right), T_{a r t}$ is the arterial temperature and $T_{\text {ven }}$ the venous one, that is usually considered equal to tissue temperature at that point. Equation (3) admits also an integraldifferential expression in the following way:

$$
\int_{V} q(\vec{r}, t) d V=\int_{V} \rho c \frac{d T(\vec{r}, t)}{d t} d V-\int_{S} k \nabla T(\vec{r}, t) \hat{n} d S-\int_{V} \rho_{b} c_{b} w_{b}\left[T_{a r t}(\vec{r}, t)-T_{v e n}(\vec{r}, t)\right] d V .
$$

Here $q(\vec{r}, t)$ is the volumetric heat generated in the $\vec{r}$ position and time $t$, originated due to the energy of the optical source. The equation is defined in a volume $\mathrm{V}$, which area is represented as $\mathrm{S}$.

The treatment of boundary conditions depends on the concrete problem we are dealing with. The most usual case is to have an extent biological tissue irradiated from the outside and heating area being only a part of that tissue. Under this 
geometry, there would be a superficial boundary in contact with air. The rest of the limiting parts of the grid would be treated so as to not influence the calculations, increasing the analysed area in case this occurs. In the upper surface in contact with air, convection, radiation and vaporization effects appear. The boundary condition can be expressed as:

$$
-k \frac{d T}{d z}=h_{c}\left(T_{e}-T_{s}\right)+\sigma \varepsilon\left(T_{e}^{4}-T_{s}^{4}\right)+h_{f g} h_{m}\left(\rho_{e}-\rho_{s a t}\right),
$$

where $h_{c}$ is the convection coefficient ( $W / m^{2} K$ ), $T_{e}$ is the environmental temperature in air, $T_{s}$ is the tissue surface temperature, $\sigma=5.696 \cdot 10^{-8} \mathrm{~W} / \mathrm{m}^{2} K^{4}$ is the Stefan-Boltzmann constant, $\varepsilon$ is the emissivity, $h_{f g}$ is the phase-change enthalpy of water at $T_{s}, h_{m}$ is the convection mass transfer coefficient $(\mathrm{m} / \mathrm{s}), \rho_{e}$ is the density of water vapour in air and $\rho_{\text {sat }}$ the density of saturated water vapour at $T_{s}$.

The resolution of the bio-heat equation provides us with temperature distributions depending on both position and exposition time. As in the optical part, the accuracy in the achievement of tissue parameters is fundamental for the model to represent the experimental reality with a reasonable amount of fidelity.

\subsection{Thermal damage model}

The thermotherapy predictive analysis can go further from temperature data obtained in the previous subsection. Due to the fact that we are dealing with medical treatment, it is necessary to assure, to a great extent, that no thermal damage is going to appear, neither on the pathological tissue nor in adjacent tissues. A method to estimate this thermal damage allows a better decision on the optical source parameters, so as to provoke a therapeutic effect without collateral harmful consequences.

Thermal damage modelling presents different approaches, like CEM $43^{\circ} \mathrm{C}$ dosimetry [11] or the so-called Arrhenius integral [12]. The first one is more appropriate for determining secure temperature ranges in tissue when this temperature is constant, while the second deals with an estimation of damage with a time varying temperature distribution. This approach is what we are looking for, and is expressed by means of an integral known as Arrhenius integral:

$$
\Omega(\tau)=\ln \left(\frac{C(0)}{C(\tau)}\right)=\int_{0}^{\tau} A \cdot e^{\left(\frac{E a}{R T}\right)} \partial t,
$$

where $\Omega(\tau)$ is thermal damage, A is the frequency factor $\left(\mathrm{s}^{-1}\right), \mathrm{E}_{\mathrm{a}}$ is the activation energy of the process $(\mathrm{J} / \mathrm{mol}), \mathrm{R}$ is the universal gas constant $(8.32 \mathrm{~J} / \mathrm{mol} \cdot \mathrm{K}), \mathrm{C}(0)$ is the percentage of living cells at the beginning of irradiation, and $\mathrm{C}(\tau)$ is the same percentage at the end of the treatment after an exposition of $\tau$ seconds. In this way, after calculating thermal damage the percentage of remaining healthy cells can be shown. The dependence on the concrete tissue under treatment is contained in $\mathrm{A}$ and $\mathrm{E}_{\mathrm{a}}$ parameters, that model the speed of cell necrosis.

The change speed of thermal damage is an interesting parameter that can also be obtained from the Arrhenius integral:

$$
\frac{d(\Omega(\tau))}{d t}=A \cdot e^{\left(\frac{E a}{R T}\right)}
$$

Sometimes it is useful also to know the so-called critical temperature, id est, temperature at which thermal damage change speed or rate equals 1 :

$$
\text { Tcrit }=\frac{E_{a}}{R \cdot \operatorname{Ln}(A)}
$$

The importance of this last parameter is related with the fact that critical temperatures of some elements are known, and so the calculation of $\mathrm{A}$ and $\mathrm{E}_{\mathrm{a}}$ parameters can be made easier. 


\section{NUMERICAL METHOD FOR SOLVING THERMO-OPTICAL MODEL}

In previous sections a thermo-optical and damage model has been exposed in detail. As it could be appreciated, it is greatly based on differential or integral-differential equations, whose resolution is going to be discussed now.

The optical part was modeled via a three dimensional Beer-Lambert law, shown in equation 1. It was stated that it provides appreciably good results in biological tissue, as long as optical parameters are correctly measured. The expression is directly computable, and in this way it can be perfectly solved in an analytical manner. The result provides the optical energy distribution in the irradiated tissue.

The thermal part presents much more complexity, because it is based on the bio-heat equation or equation 3 , that is a differential equation. The analytical solution is not affordable in the general case, and so it is necessary to solve it by means of a numerical method. Numerical methods are numerous and provide different characteristics, like accuracy or computation speed. For our purposes, we could consider mainly finite difference and finite element procedures [13]. The latter is convenient for geometries that are divided in parts, regular or not, with common properties, and the analysis is made in each of them, so as to get in a later step a valid solution. However, in our case of biological tissue the latter method of finite difference seems to be more adequate, because it transforms the differential equation into a difference equation that can be solved in an iterative way.

One usual approach when applying the numerical method to the bio-heat equation is to do it with a simple explicit one step finite difference procedure [13]. Within this context, derivatives are substituted by their Taylor expansion depending on the degree of derivation, in such a way that each point is calculated from the values of points around in the previous time step. Boundary conditions apply at points where some of these surrounding points are not included in the calculation grid. The main drawbacks of the exposed considerations are the slowness of the algorithm and the problems with accuracy near the borders or when time step is not well adjusted to the specific problems. In this way, it is common to see how a simulation of irradiation during seconds can take minutes to be carried out, and how results near the boundaries resemble a kind of reflection, due to the lack of flux boundary condition.

All these problems can be avoided by the novel numerical procedure we propose. Instead of a simple one step finite difference method, we employ a two steps modified 3D Du Fort-Frankel algorithm [14], that reduces considerable the computation time and provides a more accurate prediction of temperature in tissue. The algorithm also includes features such as adaptive mesh refinement, both in time and in space, that allow the consideration of the complete heating area, and so avoid the undesired boundary distortion effects, and also contribute to calculate temperature distributions in a reasonable CPU time with a modern PC. The application of two steps modified 3D Du Fort-Frankel method to the bioheat equation expressed in equation 3 gives:

$$
\begin{aligned}
& \Theta_{i, j, k, l+1}=\Theta_{i, j, k, l-1} \frac{1-\frac{1}{\omega_{x}}-\frac{1}{\omega_{y}}-\frac{1}{\omega_{z}}}{1+\frac{1}{\omega_{x}}+\frac{1}{\omega_{y}}+\frac{1}{\omega_{z}}}+ \\
& +\frac{\Theta_{i-1, j, k, l}+\Theta_{i+1, j, k, l}}{\omega_{x}+1+\frac{\Delta x^{2}}{\Delta y^{2}}+\frac{\Delta x^{2}}{\Delta z^{2}}+\frac{\Theta_{i, j-1, k, l}+\Theta_{i, j+1, k, l}}{\omega_{y}+1+\frac{\Delta y^{2}}{\Delta x^{2}}+\frac{\Delta y^{2}}{\Delta z^{2}}}+\frac{\Theta_{i, j, k-1, l}+\Theta_{i, j, k+1, l}}{\omega_{z}+1+\frac{\Delta z^{2}}{\Delta x^{2}}+\frac{\Delta z^{2}}{\Delta y^{2}}}+} \\
& +\frac{2 t f_{i, j, k, l} a_{2} / a_{1}}{1+\frac{1}{\omega_{x}}+\frac{1}{\omega_{y}}+\frac{1}{\omega_{z}}}-\frac{2 t\left(\Theta_{i, j, k, l}-\Theta_{a r t}\right) a_{4} / a_{1}}{1+\frac{1}{\omega_{x}}+\frac{1}{\omega_{y}}+\frac{1}{\omega_{z}}}
\end{aligned}
$$

In this equation, temperature, time and spatial coordinates are normalized through: $\Theta=T / T_{D} ; \tau=t / t_{0} ; \xi=x \sqrt{s}$; $\eta=y \sqrt{s} ; \zeta=z \sqrt{s}$. The spatial mesh position is represented in the equation by (i,j,k), and time step follows the 1 index. Parameters $\omega_{q}$ are defined by $\omega_{q}=\frac{a_{1} \Delta q^{2}}{2 t}$ with $\mathrm{q}=\mathrm{x}, \mathrm{y}, \mathrm{z} ; f_{i, j, k, l}=\exp \left(-a_{3} \zeta\right) \exp \left(-\left(\xi^{2}+\eta^{2}\right)\right)$, and constants $\mathrm{a}_{\mathrm{w}}$ are 
related with thermo-optical parameters and boundary conditions: $a_{1}=\frac{\rho c}{k t_{0} s}, a_{2}=\frac{\alpha I_{0}}{k T_{D} s}, a_{3}=\frac{\alpha}{\sqrt{s}}, a_{4}=\frac{\rho_{b} c^{w} b}{k s}$, $a_{5}=\frac{0.54 k f}{k \sqrt{s}}\left[2 g \frac{\operatorname{Pr}}{D v^{2}}\right]^{0.25}, a_{6}=\frac{\sigma \varepsilon T_{D}^{3}}{k \sqrt{s}}, a_{7}=\frac{a_{5} h_{f g}}{\rho_{a} c_{a} L e^{2 / 3} T_{D}}\left(\rho_{s a t}-\rho_{e}\right)$.

The complete thermo-optical model, as long as the thermal damage analysis, was programmed in a high level language environment with an interactive user friendly interface, which main window can be seen in figure 1 . The user is asked to give optical and thermal properties, as well as air temperature and power of the optical source, and as a result temperature and thermal distributions can be obtained in a fast and accurate way. Next section illustrates the functionality of the program by an example application.

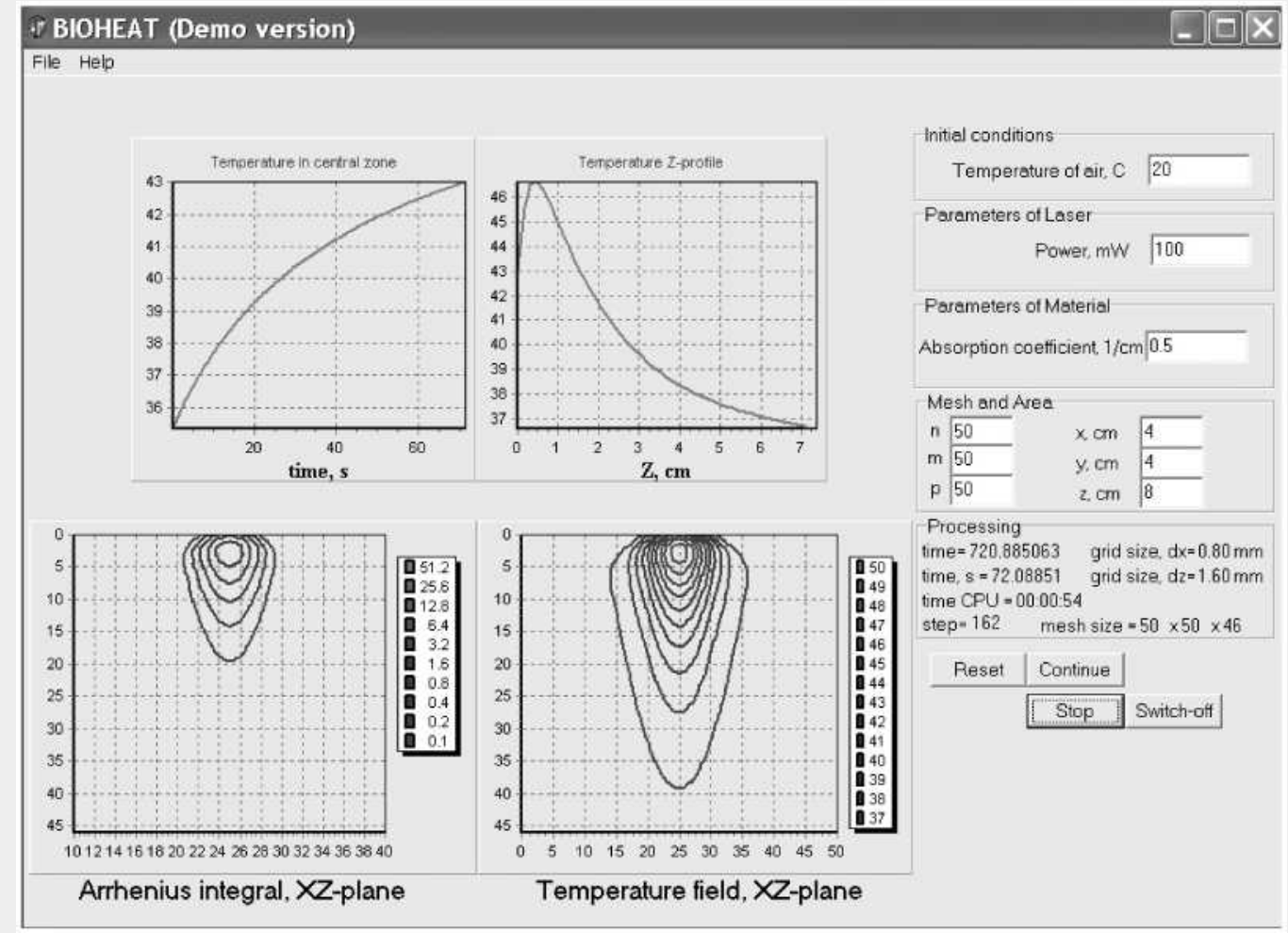

Figure 1. Capture of the main window of the program developed for implementing the thermo-optical and damage model.

\section{APPLICATION EXAMPLE RESULTS}

The program developed was applied to obtain thermal and damage distributions on bovine liver. Parameters for this sample are of relatively common use, and so the simulation can be fed with right values. Two different optical sources were considered, one with a wavelength of $1064 \mathrm{~nm}(\mathrm{Nd}: Y A G)$ and the other with $635 \mathrm{~nm}$. The absorption coefficient for the first case, that is included in the so-called therapeutic window [2], is $\alpha=0.5 \mathrm{~cm}^{-1}$, and for the other source $\alpha=2 \mathrm{~cm}^{-1}$. Some simulations were carried out and the results are presented in next figures. Attention is paid to the inclusion of several optical powers, as long as different wavelengths, positions and exposition times.

First of all, figure 2 shows the variation of temperature with depth, as a function of optical power and absorption coefficient. Power is varied from 25 to $100 \mathrm{~mW}$, with a gaussian laser beam of radius $1 \mathrm{~mm}$, and depth is scanned until 6 $\mathrm{cm}$. The different absorption coefficients used represent the wavelength variation, from the low values at $1064 \mathrm{~nm}$ to the higher at $635 \mathrm{~nm}$, out of the therapeutic window. 

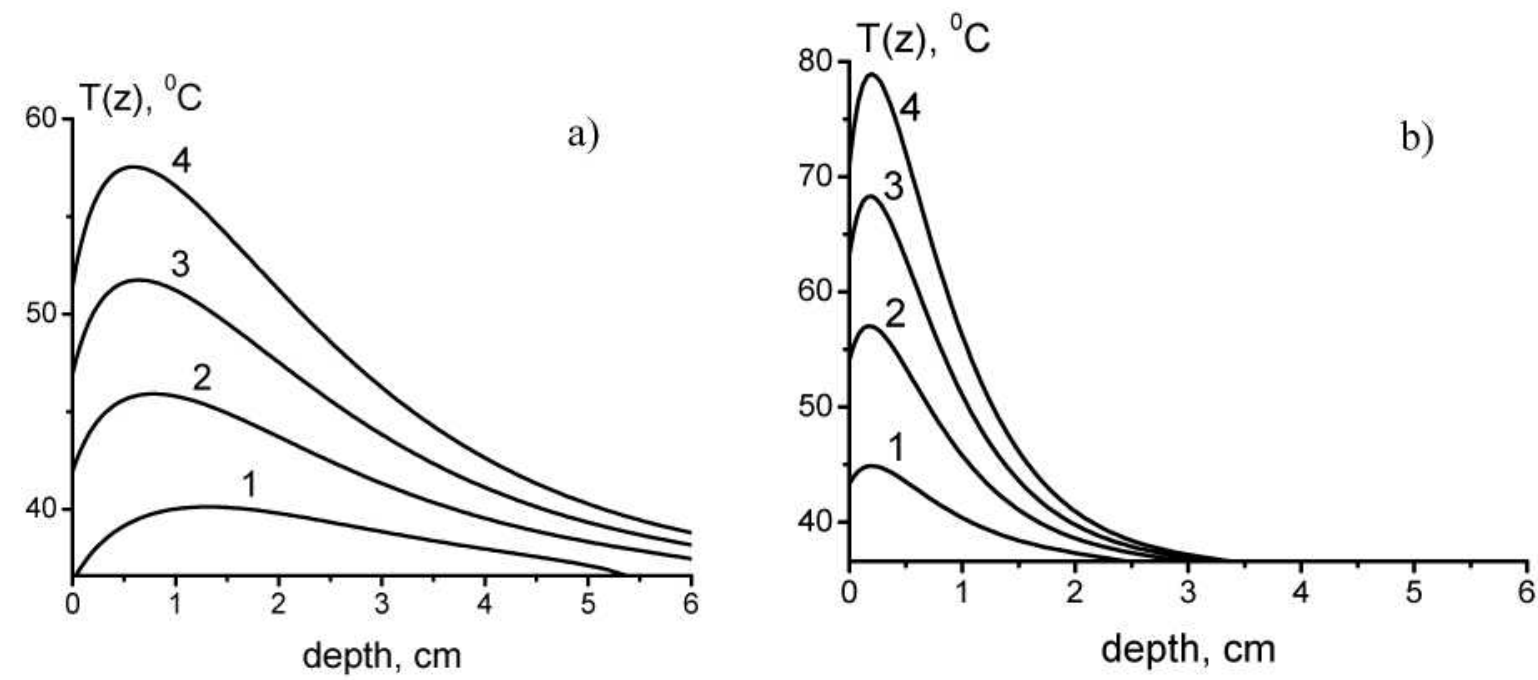

Figure 2. Temperature $\left({ }^{\circ} \mathrm{C}\right)$ profile with depth. Optical source power values are $25(1), 50(2), 75(3)$ and $100(4) \mathrm{mW}$, with a $1 \mathrm{~mm}$ radius. The absorption coefficient has a value of $0.5 \mathrm{~cm}^{-1}$ for a), and $2 \mathrm{~cm}^{-1}$ for $\mathrm{b}$ ), which correspond to a wavelength of 1064 and $635 \mathrm{~nm}$, respectively. Exposition time was increased until stabilization (10-20 minutes, depending on the optical power).

As it was expected, temperature decreases with depth, as the optical energy deposition is lower, and increasing optical power makes temperature increase. A very interesting effect of the boundary condition on the surface in contact with air establishes that the point with maximum temperature is not in the upper irradiation surface, but a few mm under it. Vaporization, along with convection and radiation, are the mechanisms actuating in this case.

Another interesting approach is to see how temperature changes in the impact point of laser source, located at $\mathrm{x}=\mathrm{y}=\mathrm{z}=0$, for different powers and wavelengths of the optical source. This simulation appears in figure 3 .
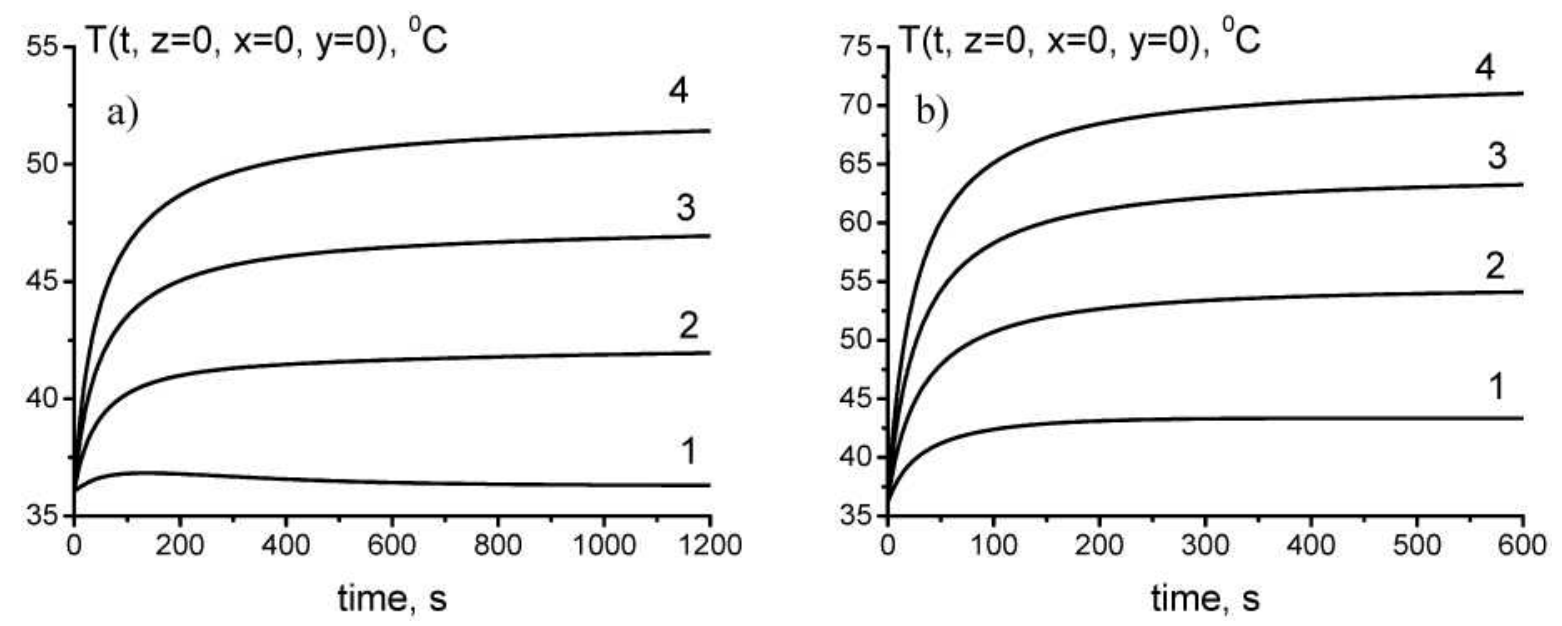

Figure 3. Temporal evolution of temperature at laser impact point $\mathrm{x}=\mathrm{y}=\mathrm{z}=0$, for different optical powers: 25 (1), 50 (2), 75 (3) and $100(4) \mathrm{mW}$. Beam radius is $1 \mathrm{~mm}$. Wavelength dependence is included by means of a changing absorption coefficient of $0.5 \mathrm{~cm}^{-1}$ for a), and $2 \mathrm{~cm}^{-1}$ for $b$ ).

From this figure 3 some comments can be made. As expected, temperature increases when optical power does. The influence of time is clear and the transient response can be appreciated due to the efficiency of the algorithm. It should 
also be mentioned that temperature tends to stabilize as time goes up, until it reaches a value that depends on the optical power of the source.

From temperature distributions in tissue, thermal damage was also calculated in two cases, with a $25 \mathrm{~mW}$ and 100 $\mathrm{mW}$ optical source. The analysis is shown in figure 4 .

a)

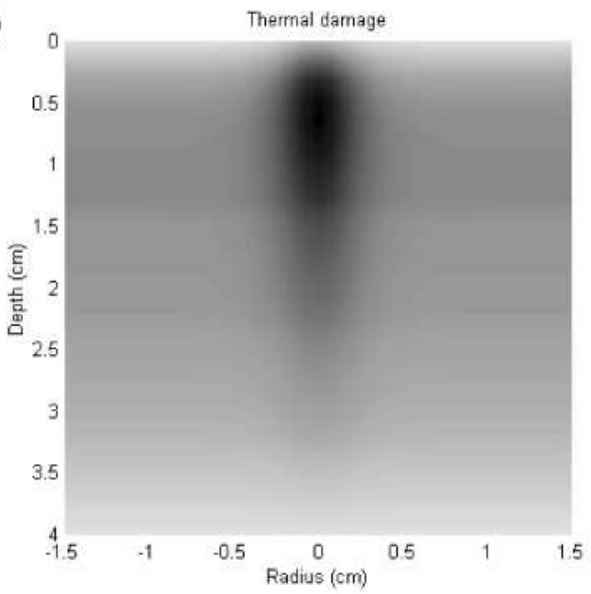

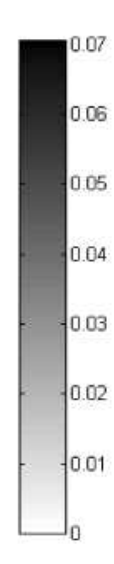

b)

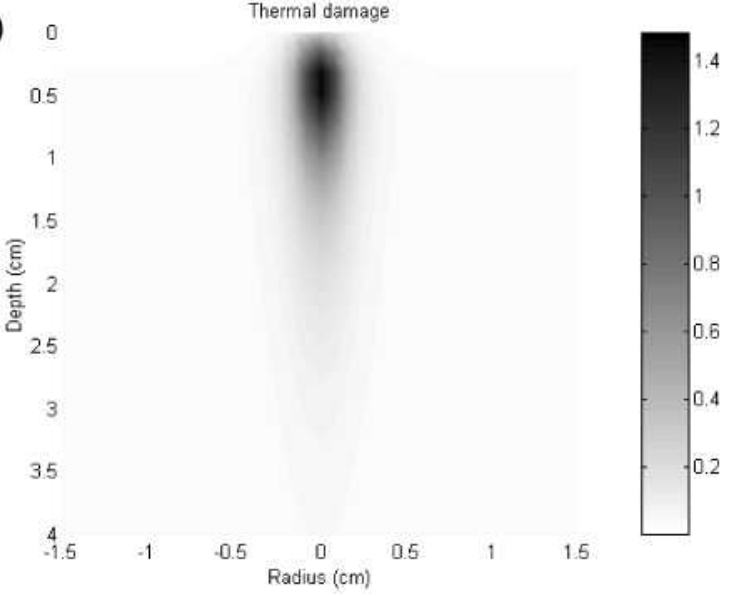

Figure 4. Thermal damage in bovine liver irradiated by a $25 \mathrm{~mW}$ (a) and a $100 \mathrm{~mW}$ (b) optical source, with a $1 \mathrm{~mm}$ beam radius, and a wavelength of $1064 \mathrm{~nm}$. Tissue was irradiated during a total of 60 seconds. Note the different scales in both graphs.

The thermal damage values are low in the case of the $25 \mathrm{~mW}$ irradiation, and become appreciable with a $100 \mathrm{~mW}$ source. Again, the worst point from the point of view of damage is not located at the upper surface, but slightly inside the tissue. From thermal damage data, the survival rate can be obtained, and in this case for the worst point the survival rate when irradiating with $25 \mathrm{~mW}$ after 60 seconds is $93.19 \%$, while in the case of $100 \mathrm{~mW}$ it decreases until $22.66 \%$. As expected, thermal damage is bigger when the optical power of the source increases.

\section{DISCUSSION}

In this article, a complete thermo-optical and damage model, programmed on a high level language environment, has been presented. The optical part has been modelled by means of a three dimensional Beer-Lambert law, thermal effects were included in a bio-heat equation and an Arrhenius analysis allowed to know the survival rates of cells in irradiated tissue. The use of a new algorithm in the solving process of bio-heat equation makes the procedure faster and more accurate. Instead of the usual explicit one step finite difference approach, a two steps modified Du Fort-Frankel algorithm was employed in the discretization of the bio-heat equation. Simulation times in a modern PC are of the order of the simulated time or even faster, depending on the specific case, and mesh dynamic spatial and temporal refinement avoids undesired boundary effects and increase the speed of the process. An application to a concrete case of bovine liver, with different wavelengths and optical source powers, as long as exposition times, was also presented to show the potentiality of the program developed.

The direct medical application of this program is quite obvious in thermotherapy, where an estimation of the temperature increase and probable thermal damage is required in order for the treatment to be as efficient as possible. Medical praxis also needs a prediction of harmful effects, either on the tissue under treatment or on adjacent ones, as for the patient not to suffer any collateral damage. With this program, the simulating process increases its speed and accuracy, so more tests could be made in the same time and therapies could be applied in a more reliable way.

\section{ACKNOWLEDGEMENTS}

This work has been carried out partially under the project TEC2006-06548/TCM of the Spanish Ministry of Education and Science. 


\section{REFERENCES}

1. D. Huang, E. A. Swanson, C. P. Lin, J. S. Shuman, W. G. Stinson, W. Chang, M. R. Hee, T. Flotte, K. Gregory, C. A. Puliafito and J. G. Fujimoto, "Optical coherence tomography", Science 254(5035), 1178-1181 (1991).

2. T. Vo-Dinh, Biomedical Photonics Handbook, CRC Press, Boca Raton, 2003.

3. T. J. Dougherty, C. J. Gomer, B. W. Henderson, G. Jori, D. Kessel, M. Korbelik, J. Moan and Q. Peng, "Photodynamic Therapy", Journal of the National Cancer Institute 90(12), 889-905, (1998).

4. M. W. Dewhirst, B. L. Viglianti, M. Lora-Michiels, M. Hanson and P. J. Hoopes, "Basic principles of thermal dosimetry and thermal thresholds for tissue damage from hyperthermia", International Journal of Hyperthermia 19(3), 267-294, (2003).

5. J. L. Arce-Diego, F. Fanjul-Vélez and A. Borragán-Torre, "Study of the thermal distribution in vocal cords irradiated by an optical source for the treatment of voice disabilities", Photonic Therapeutics and Diagnostics II: Proc. of SPIE 6078, 249-259 (2006).

6. C. F. Bohren and D. R. Huffman, Absorption and scattering of light by small particles, John Wiley \& Sons, USA, 1983.

7. G. Yoon, A. J. Welch, M. Motamedi and M. C. J. Van Gemert, "Development and application of three-dimensional light distribution model for laser irradiated tissue", IEEE Journal of Quantum Electronics 23(10), 1721-1733, (1987).

8. V. V. Barun and A. P. Ivanov, "Thermal action of a short light pulse on biological tissues", International Journal of Heat and Mass Transfer 46, 3243-3254, (2003).

9. F. P. Incropera and D. P. De Witt, Fundamentals of heat and mass transfer, Wiley \& Sons, New York, 1996.

10. H. Arkin, L. X. Xu and K. R. Holmes, "Recent developments in modelling heat transfer in blood perfused tissues", IEEE Transactions on Biomedical Engineering 41(2), 97-107, (1994).

11. M. W. Dewhirst, B. L. Viglianti, M. Lora-Michiels, M. Hanson and P. J. Hoopes, "Basic principles of thermal dosimetry and thermal thresholds for tissue damage from hyperthermia", International Journal of Hyperthermia 19(3), 267-294, (2003).

12. J. Ashley and M. Welch, Optical-thermal response of laser-irradiated tissue, Plenum Press, New York, 1995.

13. W. J. Minkowycz, E. M. Sparrow, G. E. Schneider and R. H. Pletcher, Handbook of numerical heat transfer, John Wiley \& Sons, Inc., New York, 1988.

14. V. K. Sauliev, Integration of parabolic equations by the net methods, Moscow, 1960 (in Russian). 